\title{
Interaction between paired-pulse facilitation and long-term potentiation in the projection from hippocampal area CA1 to the entorhinal cortex
}

\author{
Sarah Craig* ${ }^{*}$, Sean Commins \\ Department of Psychology, National University of Ireland, Maynooth, Co. Kildare, Ireland
}

Received 6 April 2005; accepted 15 June 2005

Available online 21 July 2005

\begin{abstract}
The hippocampus communicates with the neocortex via the entorhinal cortex and is thought to be critically involved in the consolidation of memories. This paper contains in vivo evidence of a projection from the hippocampal area CA1 to the entorhinal cortex. Current theories of memory formation suggest that the backprojections from the hippocampus to the neocortex should undergo some form of plastic change in order that memories become consolidated. Paired-pulse facilitation (PPF) and long-term potentiation (LTP) are forms of short- and long-term plasticity, respectively. We show that the CA1 to entorhinal cortex projection is capable of sustaining PPF over a wide range of stimulus intervals. In addition we demonstrate that following high frequency stimulation of this pathway the evoked response in the entorhinal cortex remains potentiated for at least 30 min. Finally, we demonstrate that PPF changes following LTP depending on the initial ratio of PPF, suggesting that LTP expression on this pathway may contain a presynaptic component. These findings should provide insight into the hippocampal function in memory formation.
\end{abstract}

(C) 2005 Elsevier Ireland Ltd and the Japan Neuroscience Society. All rights reserved.

Keywords: Long-term potentiation (LTP); Paired-pulse facilitation (PPF); Presynaptic; Hippocampus; CA1; Entorhinal cortex; Synaptic plasticity; Memory

It is widely accepted that the structures of the medial temporal lobe are involved in learning and memory. Damage to this area can lead to severe declarative memory impairments (Scoville and Milner, 1957). One medial temporal lobe structure considered to be critically involved in learning and memory is the hippocampal formation (O'Keefe and Nadel, 1978; Squire, 1992; Rolls and O'Mara, 1993; Nadel and Moscovitch, 1997). The hippocampal formation is anatomically defined by a number of subregions that include the dentate gyrus (d.g.), areas CA3, CA1 and the subiculum. Although it is believed that the hippocampus is the site of association of sensory information (Rolls, 1996), allowing for rapid storage of this information, the hippocampus itself is not thought to be involved in the long-term storage of this information (Squire

\footnotetext{
* Corresponding author. Tel.: +353 1 7086068; fax: +3531 1084767 .

E-mail address: Sarah.Craig@may.ie (S. Craig).
}

and Alvarez, 1995). Instead long-term memory storage is thought to occur in the neocortex (Squire et al., 1984; Squire, 1992; McClelland et al., 1995). Therefore, an interface between the hippocampal formation and the neocortex would be required to translate temporary hipppocampal information storage into a more permanent cortical storage.

The return projections from the hippocampus to the cortex are thought to originate exclusively from area CA1 and the subiculum, thereby making both/either of these structures potential candidates for an interface between the hippocampus proper and the neocortex. Some projections by-pass the entohinal cortex (EC) and synapse directly on perirhinal cortex (PER) or postrhinal cortex (POR) neurons (Swanson and Cowan, 1977; Deacon et al., 1983), however, the majority of backprojections to the neocortex are mainly mediated through the EC. Although both CA1 and subiculum send projections to the EC, Tamamaki and Nojyo (1995) argue that the CA1 projection is the main 
projection that feeds back information to the $\mathrm{EC}$, as many more CA1 neurons are seen to project back to the EC compared with subicular neurons.

Current theories of hippocampal function in memory propose that connections between hippocampus and neocortex undergo use-dependent changes in synaptic strength, enabling the consolidation of memory (Nadel and Moscovitch, 1997; Rolls, 1996; Graham and Hodges, 1997). Paired-pulse facilitation (PPF) and long-term potentiation (LTP) are forms of short- and long-term synaptic plasticity, respectively. PPF is the phenomenon whereby the field excitatory postsynaptic response (fEPSP) to a second stimulus is enhanced relative to the first, if the second stimulus is delivered relatively quickly after the first (Zucker, 1989). PPF is thought to have a presynaptic locus in area CA1 (Foster and McNaughton, 1991; Storm, 1992). LTP is a persistent increase in monosynaptic responsiveness induced by short high-frequency activation of the appropriate monosynaptic inputs (Bliss and Collinridge, 1993). It is a matter of controversy whether LTP is primarily a presynaptic or a postsynaptic phenomenon or some combination of the two (Bliss and Lomo, 1973). It is hypothesised that if LTP expression includes a presynaptic locus, then it might alter expression of PPF. An interaction

(a)
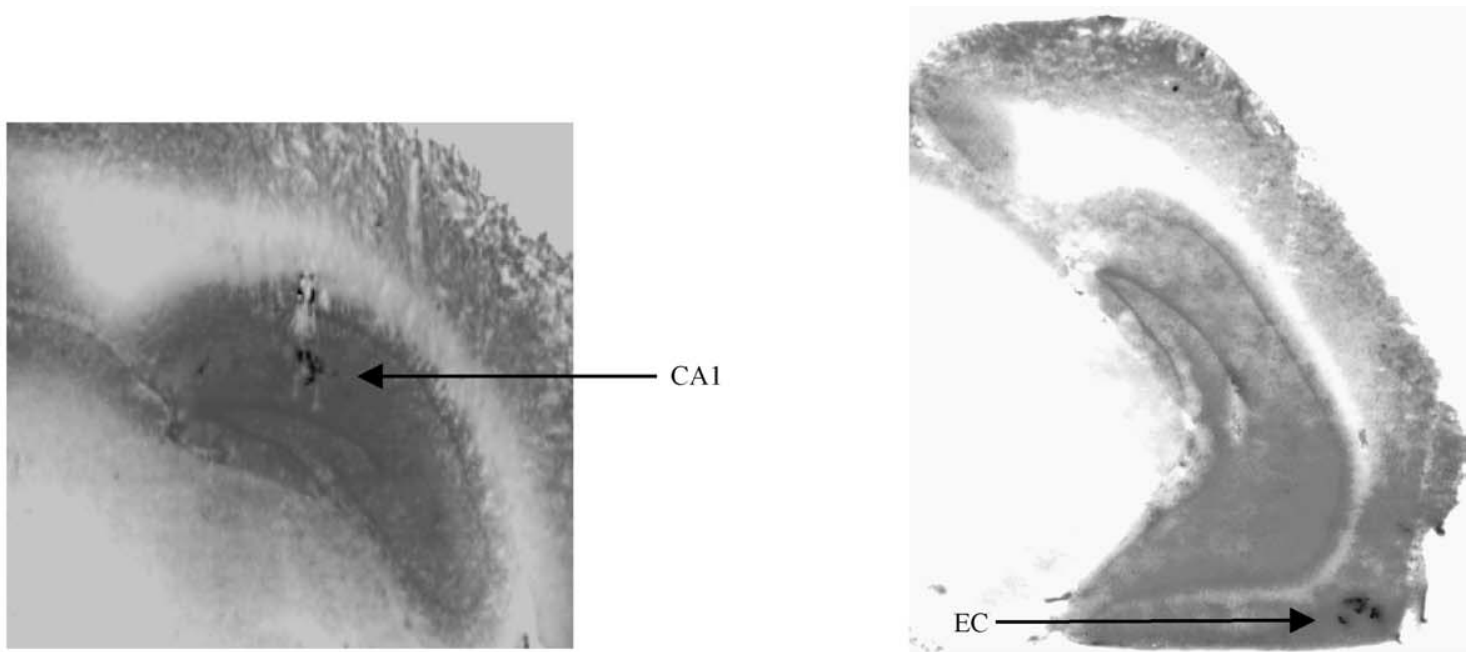

(b)
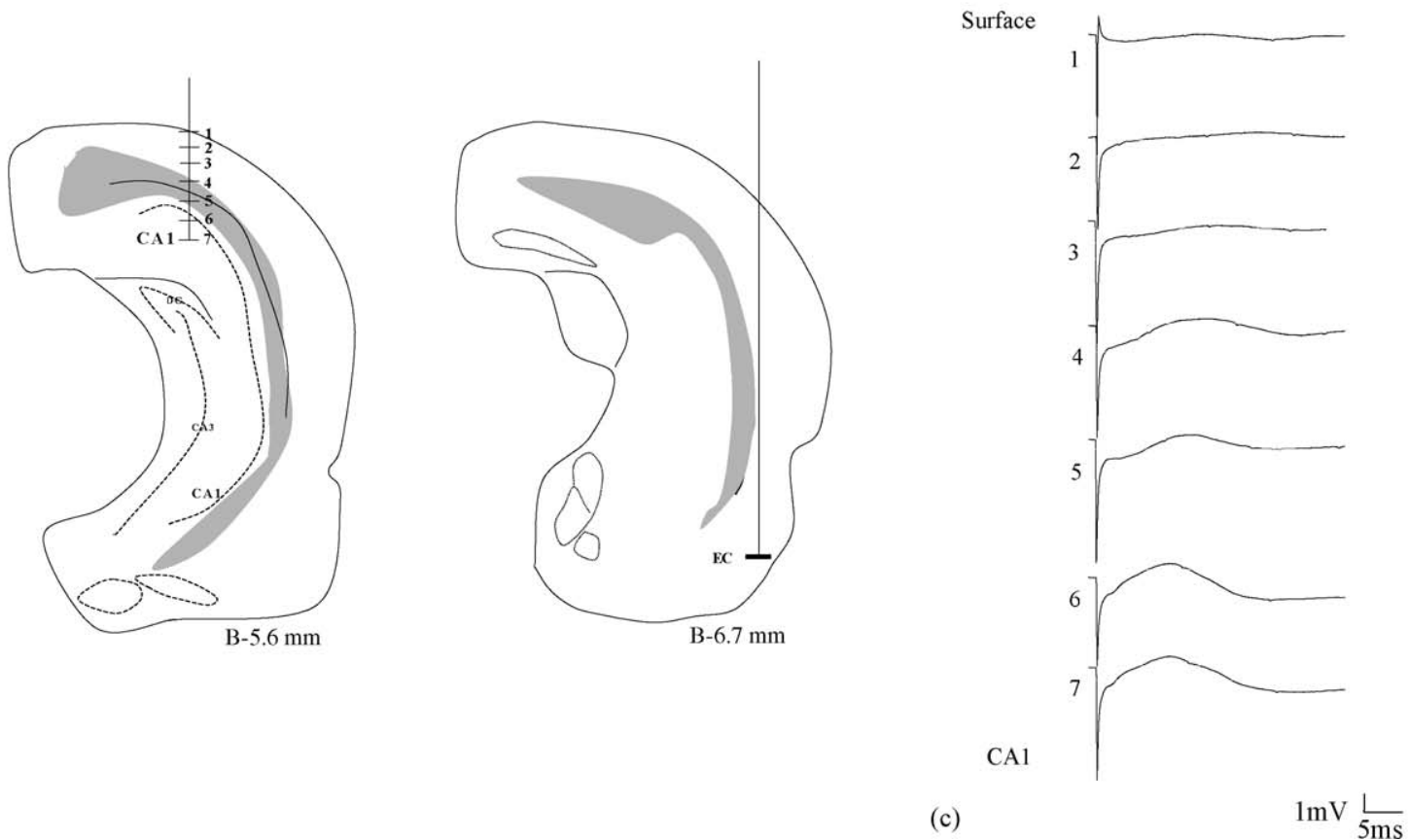

Fig. 1. Depth profile of the projection from area CA1 to the entorhinal cortex (a); Nissl-stained coronal sections, showing electrode tracks in both area CA1 and EC (b); Positions of the recording electrode in EC and seven representative positions of stimulating electrode en route to area CA1 (c); Representative depth profile of projection from area CA1 to EC, showing sample fEPSP responses at seven representative positions (1-7) from surface to CA1. 
between LTP and PPF has been previously reported in the hippocampus of rats (Commins et al., 1998a,b; Schulz et al., 1994; Christie and Abraham, 1994; Kleschevnikov et al., 1997). Commins et al. (1998a), for example, have reported that PPF decreased after LTP in the projection from CA1 to the subiculum and in the CA1 to prefrontal cortex projection PPF both increased and decreased post-LTP (Schulz et al., 1994).

In the first experiment, we examine whether there is physiological evidence to support the limited anatomical data that suggest the existence of a back projection from area CA1 to the EC. We then investigate whether this pathway is capable of sustaining both short- and long-term plasticity in the form of PPF and LTP, respectively.

Adult male Wistar rats (weight 200-300 g) were anaesthetized with urethane (ethyl carbamate: $1.5 \mathrm{~g} / \mathrm{kg}$, i.p.) and mounted on a stereotaxic holder. A local anaesthetic/adrenaline combination was injected under the scalp and an incision was made to visualize the skull. Burrholes for the stimulating and recording electrodes (stainless bipolar wires insulated except at tips; $50 \mu \mathrm{m}$ ) were placed at coordinates relative to Bregma (Paxinos and Watson, 1998): stimulating electrodes aimed at area CA1: $5.6 \mathrm{~mm}$ posterior and $4.2 \mathrm{~mm}$ lateral; recording electrodes, aimed at EC: $6.7 \mathrm{~mm}$ posterior and $5.8 \mathrm{~mm}$ lateral.

Signals were filtered between $0.1 \mathrm{~Hz}$ and $1 \mathrm{kHz}$ and then amplified. Recordings were digitised online using a PC connected to a CED-1401 plus interface (CED, Cambridge, UK). The recording electrode was slowly lowered towards the EC. The stimulating electrode was aimed at area CA1 and slowly lowered in $50 \mu \mathrm{m}$ steps. The final depth was adjusted until the maximal fEPSP was recorded. At each step two stimuli of $1 \mathrm{~mA}$ were given at a low repetition rate (interpulse interval of $20 \mathrm{~s}$ ). The first stimulus was used as a test stimulus and only the second was subsequently analysed. In all cases $(n=6)$ a response was evoked in the EC following stimulation in area CA1. Fig. 1a shows representative Nissl stained slices demonstrating the tracks of a typical stimulating and recording electrode in the CA1 and EC, respectively. Fig. $1 \mathrm{~b}$ is a schematic diagram showing the position of the recording electrode in EC and showing seven representative positions en route to area CA1. These positions correspond to the evoked responses seen in Fig. 1c. Stimulation in the overlying cortex did not produce any response in EC (see Fig. 1c: 1-3). A small positivegoing deflection occurred in EC when the corpus callosum was stimulated (Fig. 1c: 4). This evoked response gradually increased as the stimulating electrode approached the pyramidal cell layer of area CA1 (Fig. 1c: 5-7). The mean amplitude for the response at the final depth in area CA1 was $1.36 \pm 0.23 \mathrm{mV}$, with a peak latency of $11.45 \pm 0.61 \mathrm{~ms}$ (range $=9-12 \mathrm{~ms}$ ). The slope of this response was $0.23 \pm 0.05 \mathrm{mV} / \mathrm{ms}$.

PPF effects were examined after allowing both electrodes to settle for $10 \mathrm{~min}(n=5)$. Pairs of stimuli were then delivered with inter-stimulus intervals (ISIs) of 20, 40, 60,
120, 240, and $480 \mathrm{~ms}$. The first fEPSP and second fEPSP elicited by the first and second stimulus of the stimulus pair will be referred to as fEPSP1 and fEPSP2, respectively. The PPF value was calculated by taking the average of six slope values of fEPSP1, for a given ISI, and normalizing the average of six values for fEPSP2 with respect to this value in percentage terms (see Commins et al., 1998a,b). A strong PPF effect was evident with PPF appearing at the first stimulus interval $(20 \mathrm{~ms}$, fEPSP2 PPF value of $143.7 \pm 6.5 \%$ the value of fEPSP1) and was still evident at $240 \mathrm{~ms}$ (Fig. 2). A repeated measurement of ANOVA confirmed there was an overall PPF effect within groups $(F=22.327 ; \quad$ df $=1,24 ; p<0.001)$. Subsequent $t$-tests found that all paired comparisons of interest were significantly different from each other $(p<0.05)$, except for the last interstimulus interval of $480 \mathrm{~ms}$.

In a second set of experiments, we wished to determine whether the CA1 to EC pathway was capable of sustaining LTP. In addition, we wished to examine the interaction effect between PPF and LTP to determine whether plasticity in this pathway may contain a presynaptic locus. After allowing electrodes to settle in CA1 and EC, PPF was measured at two ISIs (20 and $100 \mathrm{~ms}$ ) in a further 10 animals (Fig. 3a). The first value of $20 \mathrm{~ms}$ was chosen as it represented the first interval where facilitation occurred; the second value of $100 \mathrm{~ms}$, because this was where the facilitatory effect started to diminish (as demonstrated in experiment 1; see Fig. 2). An earlier study looking at PPF between CA1 and the prefrontal cortex also observed that the maximal effect of PPF occurred around $100 \mathrm{~ms}$ (Izaki et al., 2003). A strong PPF effect was evident at both intervals $(20 \mathrm{~ms}$, fEPSP2 PPF value of $191.6 \pm 12 \%$ the value of fEPSP $1 ; 100 \mathrm{~ms}$, fEPSP 2 PPF value of $153.02 \pm 7.45 \%$ the value of fEPSP1). In both groups fEPSP2 was significantly larger than fEPSP1 $(p<0.001)$.

Induction of LTP was attempted with a high-frequency stimulation (HFS) protocol, following a $10 \mathrm{~min}$ baseline period (LFS $0.05 \mathrm{~Hz}$ ). HFS consisted of three trains of stimuli for $200 \mathrm{~ms}$ at $250 \mathrm{~Hz}$, with an inter-train interval of $30 \mathrm{~s}$ (see Gooney et al., 2002 for details). Stimulus intensity during LTP induction was set at baseline intensity. Lowfrequency stimulation was then resumed at a rate of $0.05 \mathrm{~Hz}$ for a further $30 \mathrm{~min}$. The HFS protocol used here induced robust long-term enhancement of synaptic transmission in the CA1 to EC projection. Fig. 2b demonstrates that LTP was successfully induced $(n=10)$ and that evoked responses remained potentiated for at least $30 \mathrm{~min}$. A dependent $t$-test was carried out to compare the last $10 \mathrm{~min}$ of baseline with the last 20-30 min period post-HFS for slope measurements. The period was chosen to examine the stability of the potentiated response over time. A significant degree of potentiation post-HFS relative to the baseline was observed $(t=-28.374 ; \mathrm{df}=1,28 ; p<0.001)$.

PPF was then induced $30 \mathrm{~min}$ post-HFS. There was no change in PPF after HFS compared with before HFS (data not shown). Other groups (Schulz et al., 1994; Kleschevni- 

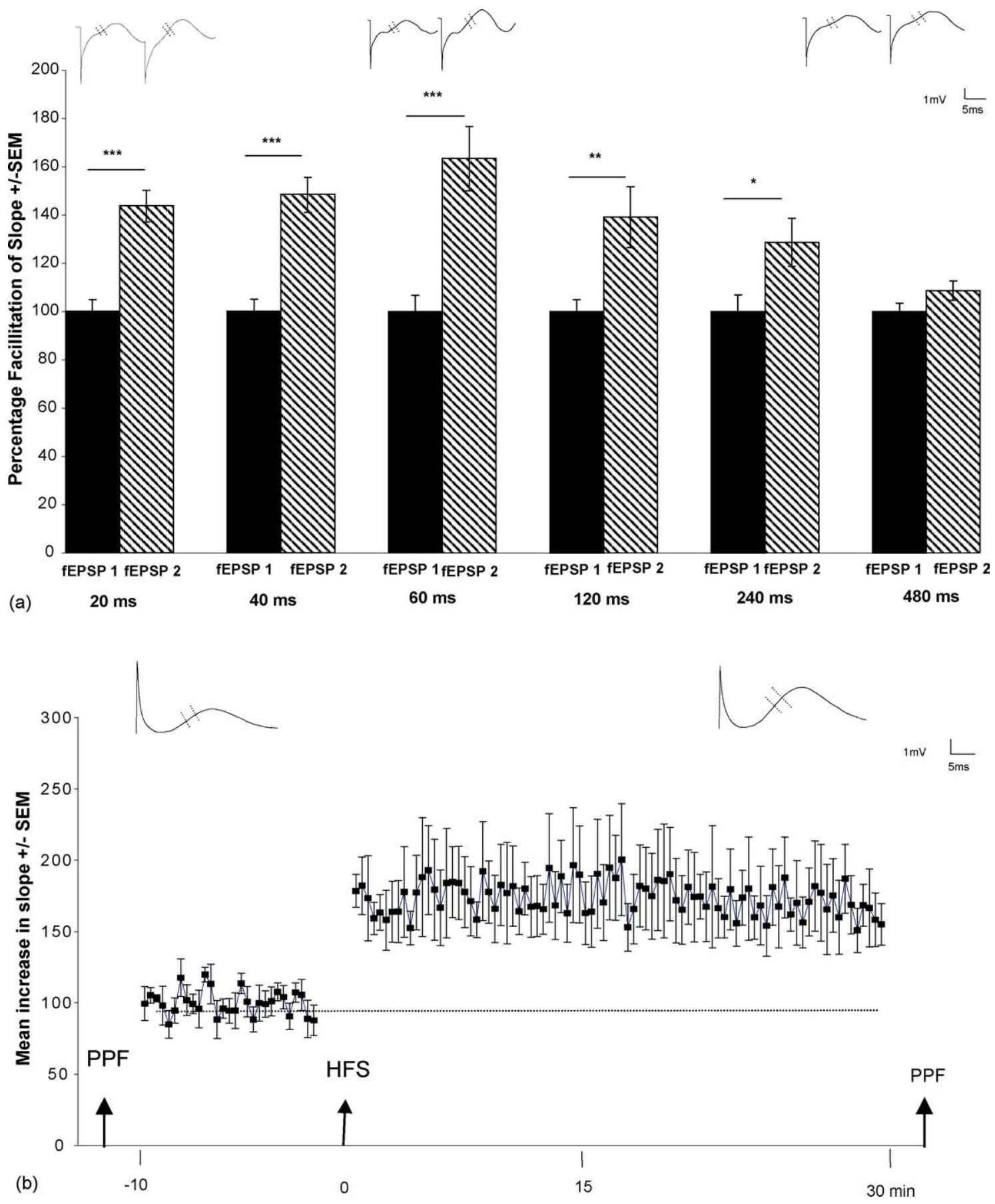

Fig. 2. Plasticity in the CA1 to EC projection (a); A bar chart showing paired-pulse facilitation in the CA1-EC pathway for intervals indicated; bars represent mean peak slopes for fEPSP1 (black) and fEPSP2 (hatched) $\left.{ }^{* * * *} p<0.001,{ }^{* * *} p<0.01,{ }^{*} p<0.05\right)$. Data are normalised to fEPSP1 (100\%). Representative traces for 20,60 and $480 \mathrm{~ms}$ intervals can be seen above graph (b); Effects of HFS on the slope of fEPSPs. The post-HFS values are expressed as percentage of the pre-stimulation baseline \pm S.E.M. Representative traces for pre- and post-HFS above graph. Time course for experiment shown along $x$-axis; PPF was induced then 10 min baseline measurements, HFS was induced, then 30 min baseline measurements, PPF was induced.

kov et al., 1997) have noticed that changes in PPF depend on the initial PPF ratio. To illustrate this fact, the data were divided into two subsets depending on whether the initial PPF ratio (for $100 \mathrm{~ms}$ ) was above (Fig. 3a) or below the mean facilitatory increase (150\%) (Fig. 3b). A significant difference was observed between these two groups for both
ISIs $(p<0.01)$. Larger LTP was also observed in the group with the larger initial PPF (198.41\%, see Fig. 3c) that was significantly different $(t=22.333, \mathrm{df}=53, p<0.001)$ to the group with the lower initial PPF (141.08\%, Fig. 3d).

A significant decrease in PPF after LTP was observed for the group with the initial large PPF (Fig. 3e, $20 \mathrm{~ms}$ : 

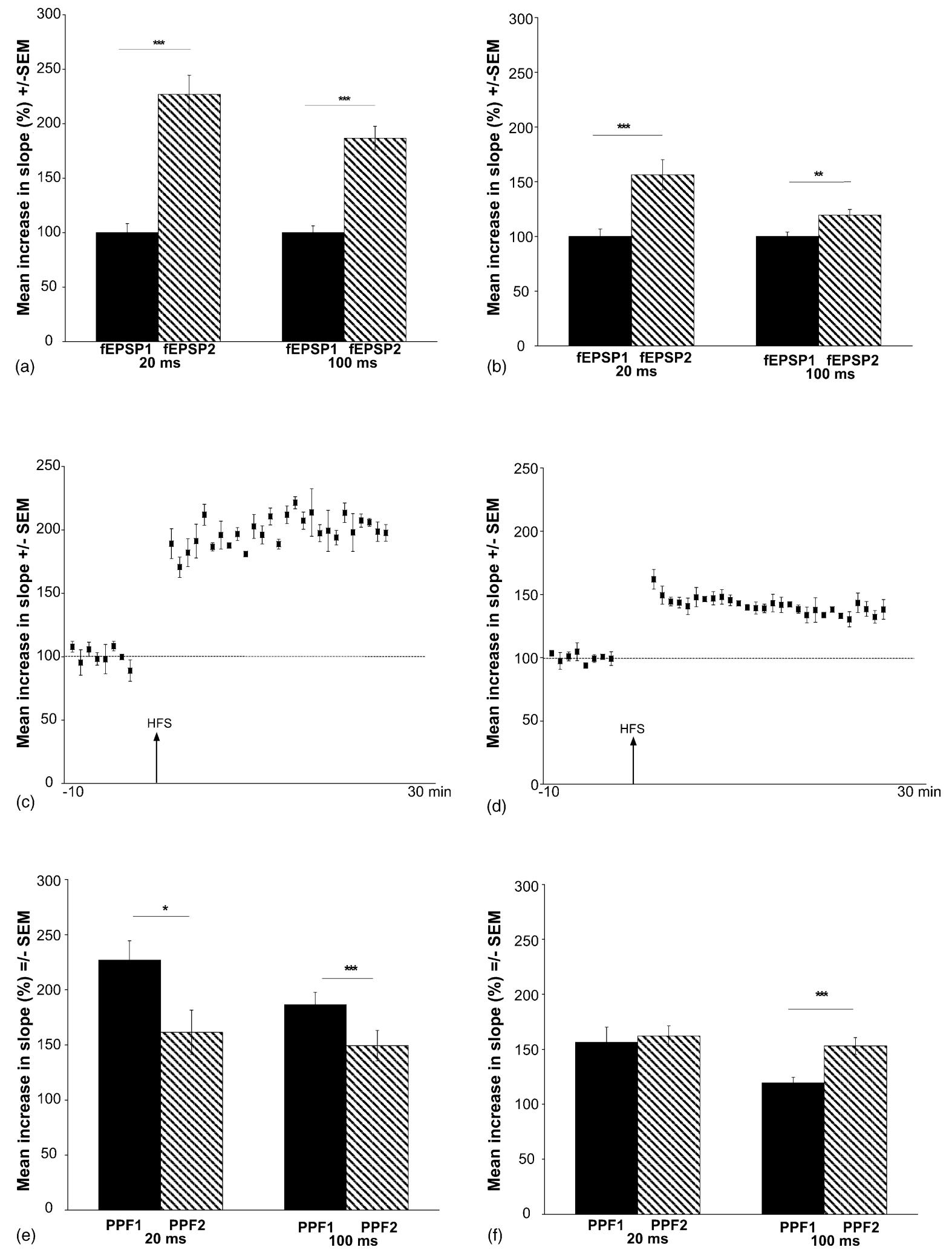

Fig. 3. Dependence of LTP (c and d) and changes in PPF ratio (e and f) on initial PPF level (a); PPF for the group with initial high PPF (over 150\%). Black bars represent fEPSP 1 and hatched bars represent fEPSP2 (b); PPF for the group with initial low PPF (below 150\%) (c); LTP for the group with initial high PPF (d); LTP for the group with initial low PPF (e); Mean PPF before (black) and after (hatched) HFS was induced for the group with initial high PPF (c); Mean PPF before and after HFS was induced for the group with initial low PPF. 
$t=2.638, \mathrm{df}=1,29 ; p<0.05 ; 100 \mathrm{~ms}: t=5.063 ; \mathrm{df}=1,29$; $p<0.001)$. Whereas, in the group with the smaller initial PPF an increase in PPF after LTP was observed at $100 \mathrm{~ms}$ (Fig. 3f, $t=-4.472$; df $=1,29 ; p<0.001$ ) with no change in PPF following $20 \mathrm{~ms}$ ISI.

This study found physiological evidence for a projection from area CA1 of the hippocampus to the entorhinal cortex. This projection is capable of undergoing both short- and long-term synaptic changes. This is the first demonstration in vivo that this output of the hippocampus is capable of undergoing such synaptic changes.

The subiculum and area CA1 are thought to act as the main output structures of the hippocampus to send hippocampally-processed information to the neocortex for storage. However, very little information regarding the projections from either of these areas is available. Anatomical studies (Tamamaki and Nojyo, 1995) proposed a projection from CA1 to EC. In this study, we have confirmed the presence of a physiologically responsive projection.

We suggest this CA1 to entorhinal cortex projection is monosynaptic in nature. Several previous studies have interpreted a short latency as consistent with monosynaptic activation (Burette et al., 1997; Cousens and Otto, 1998) and we observe similar short EPSP peak latencies (range: 9-12 ms) in this projection. Other authors (Leung, 1979; Yeckel and Berger, 1990; Cousens and Otto, 1998) suggest that following trains of continuous stimulation all components of the EPSP should remain intact for a monosynaptic response. We observed that the present responses followed $100 \mathrm{~Hz}$ stimulation (data not shown), further supporting the conclusion that this is a monosynaptic projection.

Furthermore, this projection shows PPF across nearly the full range of stimulus intervals tested. PPF maximises between 40 and $120 \mathrm{~ms}$ and declines as the ISIs are increased or decreased. These results compare favourable with results obtained from other intrinsic hippocampal projections, for example, the CA1 to subiculum projection (Commins et al., 1998a,b). In the second experiment, the magnitude of PPF values for the $20 \mathrm{~ms}$ interval was different to the first experiment. This may be due to the fact that PPF can vary dramatically from synapse to synapse (Hessler et al., 1993) or within and between slices (Schulz et al., 1995).

It has been proposed that connections between the hippocampus and the neocortex undergo use-dependent synaptic changes, enabling the consolidation of memories (Rolls, 1996; Nadel and Moscovitch, 1997; Graham and Hodges, 1997). Rolls (1996) suggests that the hippocampus operates as an intermediate-term memory and that long-term memories are laid down in the neocortex. In contrast, Nadel and Moscovitch (1997) proposed that the interaction between the hippocampus and the neocortex itself is the memory trace. However, both of these theories recognise the need for an interface between the presumed memory function of the hippocampus and the neocortex. The hippocampal output to the neocortex is mainly mediated through the EC (Burwell and Amaral, 1998). We provide evidence here for the first time, that synaptic plasticity exists in one of the main output projections from the hippocampus to EC. We were able to induce long-term changes in this pathway that lasted for at least $30 \mathrm{~min}$. This suggests that area CA1 may act as the interface between the hippocampally-processed information and the neocortex during consolidation of long-term memories. Both area CA1 and subiculum are thought to be the main output structures of the hippocampal formation. LTP has been observed on the CA1subiculum projection (Commins et al., 1998a,b, 2002) and now on the CA1 to EC projection. Further studies should examine whether the subiculum-EC projection also undergoes LTP.

Whether LTP has a pre- or postsynaptic locus is a matter of debate. To help understand LTP location, McNaughton (1982) developed an approach based on the analysis of changes in PPF following LTP. These authors suggest that PPF should change following LTP if presynaptic mechanisms contribute to LTP expression. Consistent with other studies (Schulz et al., 1994, 1995; Kleschevnikov et al., 1997) we observed a change in PPF with LTP that was dependent on the initial level of PPF. A high initial PPF lead to a decrease in PPF after LTP, whereas a low initial PPF lead to an increase in PPF after LTP. Also similar amounts of LTP were associated with similar amounts of initial PPF. It is hypothesized that PPF results from an increase in the probability of transmitter release $(p)$ during the second paired-pulse (Foster and McNaughton, 1991; Storm, 1992). Schulz et al. (1994) suggest the amplitude of an EPSP equals the number off transmitter release sites $(n)$ times the probability of release $(p)$. As a large LTP has been shown to be associated with a large initial PPF in this study and others (Schulz et al., 1994, 1995; Kleschevnikov et al., 1997; Commins et al., 1998a,b; Li et al., 2000), it is likely that LTP involves an increase in $p$. As neurotransmitter release is limited, this increase in $p$ would lead to a decrease in PPF, as there would be no more capacity for $p$ to increase (see Schulz, 1997). This would suggest a presynaptic involvement in LTP, although some additional postsynaptic involvement has not been ruled out.

To conclude, in this study we confirmed the presence of a projection from area CA1 to the entorhinal cortex. This pathway was capable of undergoing both short- and longterm synaptic changes. This suggests that area CA1 may act as a possible interface between the hippocampus and the neocortex during the consolidation of memories. We also suggest that the expression of LTP in this pathway may include a presynaptic locus.

\section{Acknowledgements}

The authors gratefully acknowledge the help of Shane O'Mara and Thelma Crowley (Trinity College) and Derek Walsh (NUI Maynooth). 


\section{References}

Bliss, T.V.P., Lomo, T., 1973. Long-lasting potentiation of synaptic transmission in the dentate area of the anaesthetized rabbit following stimulation of the perforant path. J. Physiol. (Lond.) 232, 331-356.

Bliss, T.V.P., Collinridge, G.L., 1993. A synaptic model for memory: longterm potentiation in the hippocampus. Nature 361, 31-39.

Burette, F., Jay, T.M., Laroche, S., 1997. Reversal of LTP in the hippocampal afferent fiber system to the prefrontal cortex in vivo with lowfrequency patterns of stimulation that do not produce LTD. J. Neurophysiol. 78, 1155-1160.

Burwell, R.D., Amaral, D.G., 1998. Cortical afferents of the perirhinal, postrhinal and entorhinal cortices of the rat. J. Comp. Neurol. 398, 179205.

Christie, B.R., Abraham, W.C., 1994. Differential regulation of paired-pulse plasticity following LTP in the dentate gyrus. Neuroreport 5, 385-388.

Commins, S., Gigg, J., Anderson, M., O'Mara, S.M., 1998a. Interaction between paired-pulse facilitation and long-term potentiation in the projection from hippocampal area CA1 to the subiculum. Neuroreport 9, 4109-4113.

Commins, S., Gigg, J., Anderson, M., O’Mara, S.M., 1998b. The projection from hippocampal area CA1 to the subiculum sustains long-term potentiation. Neuroreport 9, 847-850.

Commins, S., Aggleton, J.P., O’Mara, S.M., 2002. Physiological evidence for a possible projection from dorsal subiculum to hippocampal area CA1. Exp. Brain Res. 146, 155-160.

Cousens, G., Otto, T.A., 1998. Induction and transient suppression of longterm potentiation in the peri- and postrhinal cortices following thetarelated stimulation of hippocampal field CA1. Brain Res. 780, 95-101.

Deacon, T.W., Eichenbaum, H., Rosenberg, P., Eckmann, K.W., 1983. Afferent connections of the perirhinal cortex in the rat. J. Comp. Neurol. 220, 168-190.

Foster, T.C., McNaughton, B.L., 1991. Long-term enhancement of CA1 synaptic transmission is due to increased quantal size, not quantal content. Hippocampus 1, 79-91.

Gooney, M., Shaw, K., Kelly, A., Lynch, M.A., 2002. Long-term potentiation and spatial learning are associated with increased phosphorylation of TrkB and extracellular signal-regulated kinase (ERK) in the dentate gyrus: evidence for a role for brain-derived neurotrophic factor. Behav. Neurosci. 116, 455-463.

Graham, K.S., Hodges, J.R., 1997. Differentiating the roles of the hippocampal complex and the neocortex in long-term storage: evidence from the study of semantic dementia and AD. Neuropsychology 11, 1-13.

Hessler, N.A., Shirke, A.M., Malinow, R., 1993. The probability of transmitter release at a mammalian central synapse. Nature 366, 569-572.

Izaki, Y., Takita, M., Nomura, M., Akema, T., 2003. Differences between paired-pulse facilitation and long-term potentiation in the dorsal and ventral hippocampal CA1-prefrontal pathways of rats. Brain Res. 992, 142-145.

Kleschevnikov, A.M., Sokolov, M.V., Kuhnt, U., Dawe, G.S., Stephenson, J.D., Voronin, L.L., 1997. Changes in paired-pulse facilitation correlate with induction of long-term potentiation in area CA1 of rat hippocampal slices. Neuroscience 76, 829-843.

Li, S., Anwyl, R., Rowan, M.J., 2000. A persistent reduction in the shortterm facilitation accompanies long-term potentiation in the CA1 areas in the intact hippocampus. Neuroscience 100, 213-220.
Leung, L.S., 1979. Orthodromic activation of hippocampal CA1 region of the rat. Brain Res. 176, 49-63.

McClelland, J.L., McNaughton, B.L., O’Reilly, R.C., 1995. Why are there complementary learning systems in the hippocampus and neocortex: insights from the successes and failures of connectionist models of learning and memory. Psychol. Rev. 102, 419-457.

McNaughton, B.L., 1982. Long-term synaptic enhancement and short-term potentiation in rat fascia dentata act through different mechanisms. J. Physiol. (Lond.) 324, 249-262.

Nadel, L., Moscovitch, M., 1997. Memory consolidation, retrograde amnesia and the hippocampal complex. Curr. Opin. Neurobiol. 7, 217-227.

O'Keefe, J., Nadel, L., 1978. The Hippocampus as a Cognitive Map. The Clarendon Press, Oxford.

Paxinos, G., Watson, C., 1998. The Rat Brain in Stereotaxic Coordinates. Academic Press, San Diego.

Rolls, E.T., O’Mara, S.M., 1993. Neurophsyiological and theoretical analysis of how the primate hippocampus functions in memory. In: Ono, T., Squire, L.R., Raiche, M.E., Perrett, D.I., Fukuda, M. (Eds.), Brain Mechanisms of Perception: From Neuron to Behaviour. Oxford University Press, New York, pp. 276-300.

Rolls, E.T., 1996. A theory of hippocampal function in memory. Hippocampus 6, 601-620.

Schulz, P.E., Cook, E.P., Johnston, D., 1994. Changes in paired-pulse facilitation suggest presynaptic involovement in long-term potentiation. J. Neurosci. 14, 5325-5337.

Schulz, P.E., Cook, E.P., Johnston, D., 1995. Using paired-pulse facilitation to probe the mechanisms for long term potentiation (LTP). J. Physiol. (Paris) 89, 3-9.

Schulz, P.E., 1997. Long-term potentiation involves increases in the probability of neurotransmitter release. Proc. Natl. Acad. Sci. 94, 58885893.

Scoville, W.B., Milner, B., 1957. Loss of recent memory after bilateral hippocampal lesions. J. Neurol. Neurosurg. Psychiatr. 20, 11-21.

Squire, L.R., Cohen, N.J., Nadel, L., 1984. The medial temporal regions and memory consolidation: a new hypothesis. In: Weingartner, H., Parker, E. (Eds.), Memory Consolidation. Erlbaum, Hillsdale, NJ, pp. 185 210.

Squire, L.R., 1992. Memory and the hippocampus: a synthesis from findings with rats, monkeys, and humans. Psychol. Rev. 99, 195-231.

Squire, L.R., Alvarez, P., 1995. Retrograde amnesia and memory consolidation: a neurobiological perspective. Curr. Opin. Neurobiol. 5, 178 183.

Storm, J.F., 1992. Transmission-dependent depression coexists with facilitation at hippocampal excitatory synapses. Soc. Neurosci. Abstr. 18, 1340 .

Swanson, L.W., Cowan, W.M., 1977. An autoradiographic study of the organization of the efferent connections of the hippocampal formation in the rat. J. Comp. Neurol. 172, 49-84.

Tamamaki, N., Nojyo, Y., 1995. Preservation of topography in the connections between the subiculum, field CA1 and the entorhinal cortex in rats. J. Comp. Neurol. 353, 379-390.

Yeckel, M.F., Berger, T.W., 1990. Feedforward excitation of the hippocampus by afferents from the entorhinal cortex: redefinition of the role of the trisynaptic pathway. Proc. Natl. Acd. Sci. 87, 5832-5836.

Zucker, R.S., 1989. Short-term synaptic plasticity. Ann. Rev. Neurosci. 12, 13-31. 